\title{
SUBMILLIMETER STRUCTURE OF THE DISK OF THE BUTTERFLY STAR
}

\author{
S. WOLF \\ University of Kiel, Institute of Theoretical Physics and Astrophysics, Leibnizstrasse 15, 24098 Kiel, Germany; wolf@astrophysik.uni-kiel.de; \\ Max Planck Institute for Astronomy, Königstuhl 17, 69117 Heidelberg, Germany; swolf@mpia.de \\ A. Schegerer AND H. Beuther \\ Max Planck Institute for Astronomy, Königstuhl 17, 69117 Heidelberg, Germany; schegerer@mpia.de, beuther@mpia.de \\ D. L. PADGetT \\ California Institute of Technology, 1200 East California Boulevard, Mail Code 220-6, Pasadena, CA 91125; dlp@ipac.caltech.edu \\ AND \\ K. R. STAPELFELDT \\ Jet Propulsion Laboratory, California Institute of Technology, 4800 Oak Grove Drive, Pasadena, CA 91109; krs@exoplanet.jpl.nasa.gov \\ Received 2006 December 1; accepted 2008 January 8; published 2008 January 28
}

\begin{abstract}
We present a spatially resolved $894 \mu \mathrm{m}$ map of the circumstellar disk of the Butterfly Star in Taurus (IRAS $04302+2247$ ), obtained with the Submillimeter Array (SMA). The predicted and observed radial brightness profiles agree well in the outer disk region but differ in the inner region with an outer radius of $\sim 80-120$ AU. In particular, we find a local minimum of the radial brightness distribution at the center, which can be explained by an increasing density/optical depth combined with the decreasing vertical extent of the disk toward the center. Our finding indicates that young circumstellar disks can be optically thick at wavelengths as long as $894 \mu \mathrm{m}$. While earlier modeling lead to general conclusions about the global disk structure and, most importantly, evidence for grain growth in the disk (Wolf, Padgett, \& Stapelfeldt), the presented SMA observations provide more detailed constraints for the disk structure and dust grain properties in the inner, potentially planet-forming region ( $\leqslant 80$ $120 \mathrm{AU})$ versus the outer disk region $(\sim 120-300 \mathrm{AU})$.

Subject headings: circumstellar matter — planetary systems: formation -

planetary systems: protoplanetary disks - radiative transfer stars: individual (IRAS 04302+2247) — submillimeter

Online material: color figure
\end{abstract}

\section{INTRODUCTION}

IRAS $04302+2247$ is a class I protostar in the Taurus-Auriga molecular cloud complex whose equatorial plane is inclined edge-on to the line of sight (inclination $=90^{\circ} \pm 3^{\circ}$; Wolf et al. 2003, hereafter WPS03). Parallel to the increasing amount of observational constraints, such as ground-based near-infrared images and polarization maps (Lucas \& Roche 1997, 1998), near-infrared images obtained with the Hubble Space Telescope (Padgett et al. 1999), and spatially resolved $1.3 \mathrm{~mm}$ and 2.7 mm maps (WPS03), several attempts have been undertaken to model the structure and physical conditions in the circumstellar disk and envelope of this object (e.g., Lucas \& Roche 1998; WPS03; Stark et al. 2006). In particular, spatially resolved images of the circumstellar environment of the Butterfly Star, obtained in the near-infrared and millimeter wavelength range, allowed WPS03 to conclude that the grains in the envelope of this object cannot be distinguished from those of the interstellar medium, while grains have grown via coagulation by up to $2-$ 3 orders of magnitude in the much denser circumstellar disk.

The separated dust grain evolution is in agreement with the theoretical prediction of a sensitive dependence of grain growth on the location in the circumstellar environment of young (proto)stars: grain growth is expected to occur on much shorter timescales in the dense region of circumstellar disks than in the thin circumstellar envelope. For the same reason a radial dependence of the dust grain evolution in the disk itself is expected. However, the observational data presented by WPS03 did not allow them to constrain the spatial dependence of the dust grain properties in the disk.
On the basis of radiative transfer simulations, using the disk model by WPS03, we found that further insights into and constraints for the dust grain growth as the first stage of planet formation in the circumstellar disk of the Butterfly Star can be obtained with high-resolution submillimeter observations. As outlined in $\S 3$, the apparent structure of the disk is predicted to change significantly as the observing wavelength is decreased from millimeter to submillimeter wavelengths, allowing us to constrain the radial and vertical disk structure and distribution of the dust grain properties.

In this Letter we present and discuss new, spatially resolved observations of the circumstellar disk of the Butterfly Star, obtained with the Submillimeter Array ${ }^{1}$ (SMA) at $894 \mu \mathrm{m}$. The observations and data reduction are described in $\S 2$, followed by a description of the data analysis ( $(3)$ and conclusions in $\S 4$.

\section{OBSERVATIONS AND DATA REDUCTION}

IRAS $04302+2247$ was observed with the SMA (Ho et al. 2004) on 2006 January 9 using the upper and lower sidebands at $330 \mathrm{GHz}$ and $340 \mathrm{GHz}$, respectively. The phase center was R.A. $=4^{\mathrm{h}} 33^{\mathrm{m}} 16.219^{\mathrm{s}}$ and decl. $=22^{\circ} 53^{\prime} 20.29^{\prime \prime}(\mathrm{J} 2000.0)$. Both the upper and lower sidebands had a width of $2 \mathrm{GHz}$ consisting of $24 \times 256$ channels with a spectral resolution of $0.41 \mathrm{MHz}$. The angular diameter, i.e., the radial extent of the

\footnotetext{
${ }^{1}$ The Submillimeter Array is a joint project between the Smithsonian Astrophysical Observatory and the Academia Sinica Institute of Astronomy and Astrophysics and is funded by the Smithsonian Institution and the Academia Sinica.
} 
disk derived from the previous $1.36 \mathrm{~mm}$ continuum observations, amounts to $\sim 4.3^{\prime \prime}$ (WPS03). For this reason we used the extended configuration of the array with seven antennas and corresponding projected baseline lengths of 24-254 m during the observation. The resulting size of the synthesized beam is $0.67^{\prime \prime} \times 0.53^{\prime \prime}$ with a position angle of $104^{\circ}$. The weather conditions during the $6 \mathrm{hr}$ of observation of the target were stable with typical $225 \mathrm{GHz}$ zenith sky opacities of $\tau=$ 0.05-0.07 measured by the National Radio Astronomy Observatory (NRAO) tipping radiometer operated by the Caltech Submillimeter Observatory (CSO). This result corresponds to an opacity of $\tau \approx 0.23$ at $345 \mathrm{GHz}$ (Masson 1994). The radio galaxy 3C 111 was used for gain calibration, while passband calibration was done with the quasar 3C 273. The flux calibration was determined with Uranus. The SMA data were reduced and calibrated with the MIR software package (Qi 2005). The $1 \sigma$ continuum rms amounts to $3.3 \mathrm{mJy} \mathrm{beam}^{-1}$.

The resulting $894 \mu \mathrm{m}$ continuum map of the disk of the Butterfly Star is shown in Figure 1. In order to remove the potential influence of the ${ }^{13} \mathrm{CO}(3-2)$ line at $330.587 \mathrm{GHz}$ on the total flux and continuum map, we cut a band with a width of $16 \mathrm{MHz}$ around its corresponding position in the spectrum.

The $894 \mu \mathrm{m}$ continuum flux of IRAS $04302+2247$ amounts to $267 \mathrm{mJy}$ with a corresponding calibration error of $\sim 15 \%$. This flux is consistent with our predicted flux of $225 \mathrm{mJy}$. Consequently, our SMA measurements confirm the previously estimated disk mass $\left(M_{\text {disk }}=0.07 M_{\odot}\right.$; WPS03). We wish to emphasize that this flux/mass estimate does not rely on the assumption of optically thin emission but was derived from the self-consistent model by WPS03 (i.e., self-consistent with respect to the disk density distribution, dust grain parameters, and resulting disk temperature structure; see $\S 3.1$ for details).

\section{DATA ANALYSIS}

\subsection{Disk Model}

In the following we provide a brief summary of the disk model we use in the subsequent data analysis and discussions (see WPS03 for more details):

Density profile.-We assume a disk density profile as described by Shakura \& Sunyaev (1973):

$$
\rho_{\text {disk }}=\rho_{0}\left(\frac{R_{*}}{\varpi}\right)^{\alpha} \exp \left\{-\frac{1}{2}\left[\frac{z}{h(\varpi)}\right]^{2}\right\},
$$

where $\varpi$ is the radial distance from the star in the disk midplane, $R_{*}$ is the stellar radius, and $h(\varpi)$ is the disk scale height $h=h_{0}\left(\varpi / R_{*}\right)^{\beta}$. For the exponents $\alpha$ and $\beta$ that describe the radial density profile and disk flaring, we use the relation $\alpha=3(\beta-1 / 2)$, which results from viscous accretion theory (Shakura \& Sunyaev 1973).

In addition to the circumstellar disk, the model for the environment of IRAS $04302+2247$ consists of a rotating, infalling envelope. However, on the basis of the analysis of the relative contributions of the disk and envelope to the spectral energy distribution, the second component has no significant influence on the $894 \mu \mathrm{m}$ data discussed here.

Heating sources.-The main heating source of the circumstellar environment is the embedded star. We assume typical parameters of a T Tauri star: $R_{*}=2 R_{\odot}$ and $T_{*}=4000 \mathrm{~K}$ (Gullbring 1998), which correspond to a luminosity of $L_{*}=$ $0.92 L_{\odot}$ under the assumption of a blackbody. Further heating

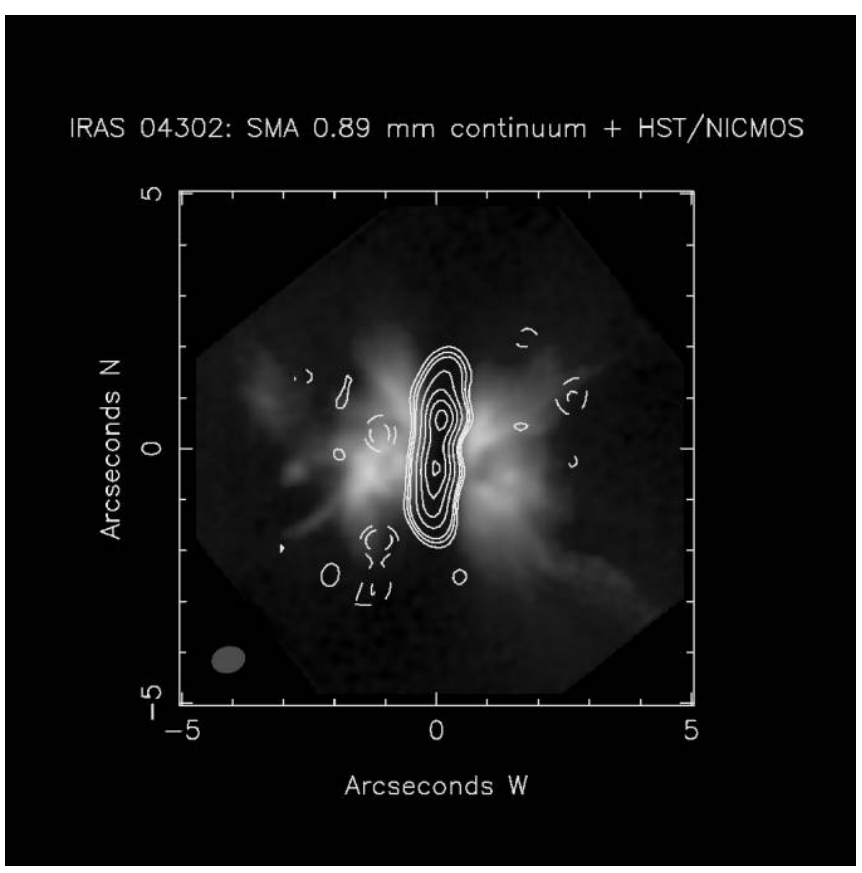

FIG. 1.-Submillimeter map of the Butterfly Star $(894 \mu \mathrm{m}$, contour lines $)$, overlaid on the near-infrared scattered-light map (Padgett et al. 1999). The levels of the solid contour are 6.8, 10.2, 13.7, 23.9, 34.1, 44.3, and $54.5 \mathrm{mJy}$ beam $^{-1}$, which correspond to the $2 \sigma, 3 \sigma, 4 \sigma, 7 \sigma, 10 \sigma, 13 \sigma$, and $16 \sigma$ levels. The levels of the dashed contours are -6.8 and -10.2 mJy beam $^{-1}$, which correspond to the $-2 \sigma$ and $-3 \sigma$ levels, respectively. [See the electronic edition of the Journal for a color version of this figure.]

of the disk is provided by accretion, where we apply the viscous disk model by Lynden-Bell \& Pringle (1974).

Dust grain properties.-We consider the dust grains to be homogeneous spheres (radius $a$ ) with a size distribution described by a power law of the form $n(a) \propto a^{-3.5}$. The dust grain ensemble consists of silicate and graphite grains with relative abundances of $62.5 \%$ astronomical silicate and $37.5 \%$ graphite. We use the optical data of "smoothed astronomical silicate" and graphite published by Weingartner \& Draine (2001) and Draine \& Lee (1984). We assume a gas-to-dust mass ratio of 100:1 and a grain mass density of $2.5 \mathrm{~g} \mathrm{~cm}^{-3}$.

Results.-Radiative transfer simulations are used to calculate the disk temperature structure self-consistently and to derive observables subsequently. These simulations are performed with the three-dimensional continuum radiative transfer code MC3D (e.g., Wolf et al. 1999; Wolf 2003). On the basis of this model, WPS03 find the following best-fit model parameters: the outer radius amounts to $300 \mathrm{AU}, \beta=58 / 45 \quad(\alpha=$ $213 / 90), h(100 \mathrm{AU})=15 \mathrm{AU}$, and $M_{\mathrm{disk}}=0.07 M_{\odot}$. In particular, an upper grain size of $\sim 100 \mu \mathrm{m}$ was found.

\subsection{Comparison of the Model Predictions with the Submillimeter Observation}

In Figure 2 the radial brightness profile along the disk midplane is shown (solid line). It agrees well with the predicted profile (dashed line) in the outer regions of the disk with radial distances larger than $~ 80-120$ AU from the center. Thus, our observations confirm the radial density distribution and dust grain properties for the outer, cold region of the disk. Consequently, our observations confirm the disk size (radius $300 \mathrm{AU}$ ) derived by WPS03.

Inside $\sim 80-120 \mathrm{AU}$ we find a discrepancy between the 


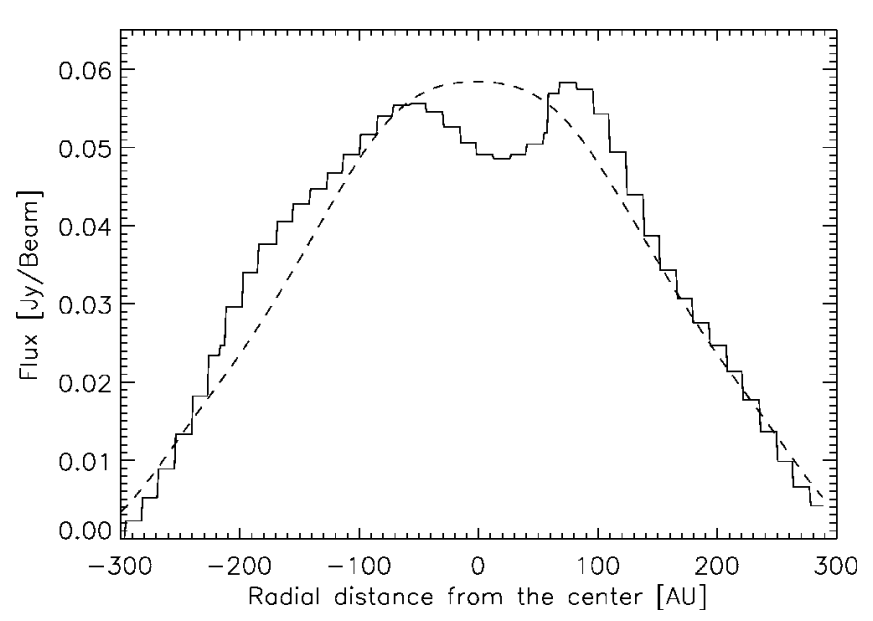

FIG. 2.-Radial brightness profile. Solid line: Observed $894 \mu \mathrm{m}$ profile. The maxima to the left and right of center are $\sim 1.4 \sigma$ and $\sim 1.8 \sigma$ above the local minimum, respectively ( $\sigma$ : standard deviation). The radial scale is based on an assumed distance of $140 \mathrm{pc}$. Dashed line: For comparison: simulated 894 $\mu \mathrm{m}$ profile, convolved with the corresponding synthesized SMA beam (based on the model by WPS03). For comparison with the $1.3 \mathrm{~mm}$ and $2.7 \mathrm{~mm}$ radial brightness profiles, see Fig. 3 in WPS03.

model prediction and the observed brightness profile. While the model predicts a rather flat plateau for this region, the observed map even shows a local minimum at the potential location of the star. The discrepancy amounts to only $\sim 2 \sigma$. However, we want to stress that the location of this minimum is not arbitrary but at the disk center. Furthermore, the corresponding local maxima show a slight symmetry with respect to the disk center.

Two explanations for this brightness minimum are at hand: First, the minimum could be caused by the lack of emitting dust, i.e., an inner region void of small dust grains. Inner holes in circumstellar disks in different stages of their evolution have been inferred from their mid-infrared spectral energy distribution and spatially resolved images. In the particular case of young disks (as the counterpart to evolved, debris-type disks), inner disk radii that are much larger than the dust sublimation radius have been deduced from the mid-infrared spectral energy distribution, for example, in the case of TW Hydrae (Calvet et al. 2002) and GM Aurigae (Rice et al. 2003). As radiative transfer simulations based on the disk model by WPS03 show, the radius of the dust-free region would have to amount to $\sim 70$ AU (compared to a radius of $\sim 300$ AU of the disk). We can test the hypothesis of the existence of a large inner hole by comparing the corresponding $1.36 \mathrm{~mm}$ simulated images with our continuum observations obtained at this wavelength with the Owens Valley Radio Observatory (OVRO; WPSO3). We find that this gap would have caused a similar local minimum at $1.36 \mathrm{~mm}$ (note that the size of the synthesized beam was similar to that of our SMA observations: $\left.0.64^{\prime \prime} \times 0.52^{\prime \prime}\right)$. Since this was not observed (see Fig. 3 in WPS03), we can exclude this explanation.

One usually would apply a second independent test to the above conclusion. If considering the disk alone, the amount of the optical to mid-infrared flux depends sensitively on the existence or absence of such a large inner hole. However, this test is not applicable in our case because the emission of the Butterfly Star is dominated by the circumstellar envelope up to wavelengths of $\sim 174 \mu \mathrm{m}$ (WPS03).

The second and thus remaining explanation is the lack of "visible" emitting dust grains. In particular, the model predicts an optical depth in the midplane (as seen from the star) that amounts to $\tau_{894 \mu \mathrm{m}} \approx 2 \times 10^{3}$. Going from millimeter to submillimeter wavelengths, the dust in the innermost, dense regions close to the disk midplane cannot be traced anymore. Consequently, the disk brightness profile that is very steep in the millimeter range becomes significantly flatter at shorter wavelengths. In other words, due to the increasing optical depth in the disk midplane, the relative contribution of the lower density outer regions and the directly heated-and therefore warm-upper disk layers (disk photosphere above the effective disk surface at $\tau_{\text {optical }} \sim 1$ ) increases with respect to the contribution from the disk midplane. This effect is amplified by the decrease of the disk scale height toward the star, resulting in a further decrease of the amount of dust in the upper, optically thin disk layers. The relative amount of dust that can efficiently contribute to the disk reemission decreases toward the star compared to the total amount of dust at the same line of sight. Depending on the optical properties of the dust grains and the particular disk structure, the radial intensity profile might thus even decrease, resulting in a local minimum at the position of the central star. The amount of dust grains that efficiently contribute to the flux measured at $1.36 \mathrm{~mm}$ (OVRO), but not at $894 \mu \mathrm{m}$ (SMA), depends sensitively both on the radial and vertical distributions of the disk density and dust grain properties and thus also on the disk temperature structure.

On the basis of the WPS03 disk model outlined in $\S 3.1$, the predicted size of the region around the star in which the flux decreases is too narrow to be spatially resolved by our SMA observations; i.e., the quantitative behavior of the radial brightness profile is different in the simulation and observation for the inner 80-120 AU (see Fig. 2). Consequently, the opacity structure, and therefore the quantities that determine it (outlined above), are different in this region than assumed in the model. As we assume perfect mixing of the dust throughout the entire disk, i.e., the dust properties and the gas-to-dust mass ratio are the same at every point in the disk, this finding is not surprising. Indeed, detailed theoretical investigations of the planet-forming process, which is expected to take place in this inner region, predict that these assumptions have to be given up because of the radial and vertical dependence of the evolution of the dust and disk parameters (e.g., Nomura \& Nakagawa 2006). However, a self-consistent model that takes into account the various processes involved (e.g., grain growth and fragmentation, dust sedimentation, radial and vertical mixing; see $\S 4$ ) is beyond the scope of this publication.

A very clear test of our conclusions will be possible with the Atacama Large Millimeter Array (ALMA; Wootten 2003). Thanks to its significantly higher spatial resolution, it will be able not only to trace the local minimum centered on the stellar position but also to detect the brightness decrease in the vertical direction, centered on the disk midplane. The resulting quadrupolar disk structure can be seen in the predicted ideal 0.3 $\mathrm{mm}, 1.36 \mathrm{~mm}$, and $2.74 \mathrm{~mm}$ maps (Fig. 4, WPS03). However, since the optical depth effect is even stronger than predicted, the effect will be even more pronounced for a given wavelength.

\section{SUMMARY AND CONCLUSIONS}

We obtained the first spatially resolved submillimeter map of the circumstellar disk of the prominent Butterfly Star in Taurus. We find a good agreement between the observed and 
the predicted brightness profiles in the outer region of the disk. However, a discrepancy between the predicted and observed radial flux distributions was found in the inner region (inside 80-120 AU), showing a decrease of the flux toward the center. This discrepancy - a local minimum at the stellar positionamounts to only $\sim 2 \sigma$. However, the specific location of the minimum at the disk center and the slight symmetry of the corresponding local maxima with respect to the disk center indicate that this profile is an intrinsic feature of the real disk brightness distribution. On the basis of radiative transfer simulations and additional observations at $1.36 \mathrm{~mm}$, we exclude a large inner hole (strongly depleted from small dust grains) as a possible explanation of this local minimum in the radial brightness distribution.

These observations may provide the basis for a detailed model of the inner structure of the disk of the Butterfly Star. While earlier modeling lead to conclusions about grain growth in the disk (WPS03), the new SMA observations provide constraints for the disk structure and dust grain properties in the inner, potentially planet-forming region ( 80-120 AU) versus the outer disk region $(\sim 120-300$ AU).

It was found that the optical depth in the inner disk region is higher than predicted by WPS03. The column density of dust along the line of sight is therefore higher in the inner disk region than derived from the previous disk model in which a perfect mixing of dust and gas throughout the entire disk is assumed. These observations indicate that there exists a higher dust density and therefore a higher dust-to-gas mass ratio in the inner disk region. This conclusion is based on the assumption that the density profile of the gas phase of the disk can be described by the same approach in the inner and outer disk regions (eq. [1]) and that there exists no discontinuity in the gas density profile.

Since the optical depth effect discussed above is constrained by both the radial and vertical disk structure and dust grain properties, such a model will have to take into account the predicted grain evolution and its dependence on the radial and vertical positions in the disk. Furthermore, dust settling and the resulting increase of the grain-grain interaction probability will result in a further vertical dependence of the grain size distribution (e.g., Weidenschilling 1997). Besides the grain evolution, mixing processes, such as convection and radial mixing within circumstellar disks, have to be considered since these processes lead to a redistribution of processed, evolved dust grains to outer, less dense, and colder disk regions (see, e.g., Klahr et al. 1999; Gail 2001).

Our observations illustrate the high potential of submillimeter observations for studying circumstellar disks around young (proto)stars. Although the spatial resolution is significantly lower than that aimed for in the case of ALMA in a few years from now, the tight correlation between the density and temperature structure in the disk is already able to constrain the radial and vertical disk structure and thus the dust grain properties as a function of the distance from the disk midplane, on the basis of the spatially resolved radial brightness profile obtained with the SMA in the submillimeter wavelength range.

S. W. and A. S. are supported by the German Research Foundation (DFG) through the Emmy Noether grant WO 857/ 2. H. B. is supported by the DFG through the Emmy Noether grant BE 2578. We wish to thank the anonymous referee for valuable suggestions concerning the presentation and discussion of our results.

\section{REFERENCES}

Calvet, N., D’Alessio, P., Hartmann, L., Wilner, D., Walsh, A., \& Sitko, M. 2002, ApJ, 568, 1008

Draine, B. T., \& Lee, H. M. 1984, ApJ, 285, 89

Gail, H.-P. 2001, A\&A, 378, 192

Gullbring, E., Hartmann, L., Briceño, C., \& Calvet, N. 1998, ApJ, 492, 323

Ho, P. T. P., Moran, J. M., \& Lo, K. Y. 2004, ApJ, 616, L1

Klahr, H. H., Henning, Th., \& Kley, W. 1999, ApJ, 514, 325

Lucas, P. W., \& Roche, P. F. 1997, MNRAS, 286, 895

- 1998, MNRAS, 299, 699

Lynden-Bell, D., \& Pringle, J. E. 1974, MNRAS, 168, 603

Masson, C. R. 1994, in ASP Conf. Ser. 59, Atmospheric Effects and Calibration, ed. M. Ishiguro \& W. J. Welch (San Francisco: ASP), 87

Nomura, H., \& Nakagawa, Y. 2006, ApJ, 640, 1099

Padgett, D. L., Brandner, W., Stapelfeldt, K. R., Strom, S. E., Terebey, S., \& Koerner, D. 1999, ApJ, 117, 1490
Qi, C. 2005, The MIR Cookbook, The Submillimeter Array/Harvard-Smithsonian Center for Astrophysics, http://cfa-www.harvard.edu/ cqi/mircook .html

Rice, W. K. M., Wood, K., Armitage, P. J., Whitney, B. A., \& Bjorkman, J. E. 2003, MNRAS, 342, 79

Shakura, N. I., \& Sunyaev, R. A. 1973, ApJ, 24, 337

Stark, D. P., Whitney, B. A., Stassun, K., \& Wood, K. 2006, ApJ, 649, 900

Weidenschilling, S. J. 1997, Icarus, 127, 290

Weingartner, J. C., \& Draine, B. T. 2001, ApJ, 548, 296

Wolf, S. 2003, Comput. Phys. Commun., 150, 99

Wolf, S., Henning, Th., \& Stecklum, B. 1999, A\&A, 349, 839

Wolf, S., Padgett, D. L., \& Stapelfeldt, K. R. 2003, ApJ, 588, 373 (WPS03)

Wootten, A. 2003, Proc. SPIE, 4837, 110 